TRANSACTIONS OF THE

AMERICAN MATHEMATICAL SOCIETY

Volume 352, Number 9 , Pages 4355-4369

S 0002-9947(00)02474-0

Article electronically published on May 12, 2000

\title{
SPECIALIZATIONS OF BRAUER CLASSES OVER ALGEBRAIC FUNCTION FIELDS
}

\author{
BURTON FEIN AND MURRAY SCHACHER
}

\begin{abstract}
Let $F$ be either a number field or a field finitely generated of transcendence degree $\geq 1$ over a Hilbertian field of characteristic 0 , let $F(t)$ be the rational function field in one variable over $F$, and let $\alpha \in \operatorname{Br}(F(t))$. It is known that there exist infinitely many $a \in F$ such that the specialization $t \rightarrow a$ induces a specialization $\alpha \rightarrow \bar{\alpha} \in \operatorname{Br}(F)$, where $\bar{\alpha}$ has exponent equal to that of $\alpha$. Now let $K$ be a finite extension of $F(t)$ and let $\beta=\operatorname{res}_{K / F(t)}(\alpha)$. We give sufficient conditions on $\alpha$ and $K$ for there to exist infinitely many $a \in F$ such that the specialization $t \rightarrow a$ has an extension to $K$ inducing a specialization $\beta \rightarrow \bar{\beta} \in \operatorname{Br}(\bar{K}), \bar{K}$ the residue field of $K$, where $\bar{\beta}$ has exponent equal to that of $\beta$. We also give examples to show that, in general, such $a \in F$ need not exist.
\end{abstract}

\section{INTRODUCTION}

Let $F$ be a field of characteristic $p \geq 0$ and let $F(t)$ denote the rational function field in one variable over $F$. Recall that $F$ is called Hilbertian if, for any irreducible polynomial $f(t, x) \in F(t)[x]$, there exist infinitely many $a \in F$ such that the specialization $t \rightarrow a \in F$ is defined on $f(t, x)$ and the specialized polynomial $f(a, x)$ is irreducible as a polynomial in $F[x]$. Global fields are Hilbertian, as are finitely generated extensions of transcendence degree $\geq 1$ of an arbitrary field (e.g., [FJ, p. 155]). In FSS, the authors and David Saltman introduced the following Brauer group analog of this property. If $p>0$, let $\operatorname{Br}(F(t))^{\prime}$ denote the subgroup of the Brauer group $\operatorname{Br}(F(t))$ of $F(t)$ consisting of all elements of order prime to $p$; if $F$ has characteristic 0 , set $\operatorname{Br}(F(t))^{\prime}=\operatorname{Br}(F(t))$. We defined $F$ to be Brauer-Hilbertian if for every element $\alpha \in \operatorname{Br}(F(t))^{\prime}$, there exist infinitely many $a \in F$ such that the specialization $t \rightarrow a$ induces a specialization $\alpha \rightarrow \bar{\alpha} \in \operatorname{Br}(F)^{\prime}$ where $\bar{\alpha}$ has exponent equal to that of $\alpha$. (The restriction to elements of order prime to $p$ was forced by our lack of understanding of the $p$-primary component of $\operatorname{Br}(F(t))$; we still do not know whether our results are valid for that component.) We showed that global fields and fields finitely generated of transcendence degree $\geq 1$ over a Hilbertian field are Brauer-Hilbertian. The result for global fields had been previously obtained by Voronovich [V] in the Russian literature; moreover, Serre [Se2] showed

Received by the editors March 23, 1998 and, in revised form, October 30, 1998.

2000 Mathematics Subject Classification. Primary 12E15, 12G05, 16K50.

Key words and phrases. Brauer group, Brauer-Hilbertian, corestriction, Hilbertian, specializations.

The authors are grateful for support under NSA Grants MDA904-97-1-0040 and MDA904-971-0060, respectively. 
that for $F$ a number field, "most" specializations into the ring of integers of $F$ preserve the order of elements of $B(F(t))$ of order 2 . Finally, we should mention that there are many examples of fields $F$ that are not Brauer-Hilbertian; these include such reasonably well-behaved fields as the field of $p$-adic numbers and the rational function field in one variable over that field (Corollary 10 and Proposition 11 of [FS2]).

Let the context be as above and let $K$ be a finite algebraic extension of $F(t)$. In this paper we will be concerned with the natural analog of the Brauer-Hilbertian property for $K$. For ease of exposition we will restrict our discussion to fields $F$ of characteristic 0 . The modifications that are needed in the general case are discussed thoroughly in [FSS]. Using those modifications, one can show that the results of this paper remain valid if one assumes that $K$ is separable over $F(t)$ and the Brauer classes considered have orders prime to the characteristic of $F$. In view of the results discussed above, it is natural to assume that $F$ is either a number field or is finitely generated of transcendence degree $\geq 1$ over a Hilbertian field of characteristic 0 ; we will make this assumption throughout. In contrast to the situation for $F(t)$, where a complete description of the structure of $\operatorname{Br}(F(t))$ is known by the AuslanderBrumer-Faddeev Theorem (e.g. [FS1, p. 51]), very little is known, in general, of the structure of $\operatorname{Br}(K)$. Because of this lack of understanding, we restrict our attention to those elements of $\operatorname{Br}(K)$ lying in the image of the restriction map, $\operatorname{res}_{K / F(t)}$, from $\operatorname{Br}(F(t))$ to $\operatorname{Br}(K)$. Our basic question, vaguely worded, is the following. Let $K$ and $F$ be as above, let $\alpha \in \operatorname{Br}(F(t))$, and let $\beta=\operatorname{res}_{K / F(t)}(\alpha)$. Do there exist infinitely many $c \in F$ such that the specialization $t \rightarrow c$ has an extension to $K$ which induces a specialization $\beta \rightarrow \bar{\beta} \in \operatorname{Br}(\bar{K}), \bar{K}$ the residue field of $K$, where $\bar{\beta}$ has exponent equal to that of $\beta$ ? Even under all of these restrictions, it turns out that, in general, the answer to our question is "no". Our main theorem, however, provides an easily checkable finite set of sufficient conditions for an affirmative answer. In particular, we obtain easily verifiable conditions on $\alpha \in \operatorname{Br}(F(t))$ so that $\operatorname{res}_{K / F(t)}(\alpha) \neq 0$. In the last portion of the paper, we present three examples to illustrate the various possibilities when the hypotheses of our main theorem are not satisfied.

\section{Notation, Definitions, and Preliminaries}

We begin by establishing some of the notation that will be used throughout this paper. In general, we will follow the terminology of [FSS]. In what follows $L / E$ will always be an extension of fields.

We denote the algebraic closure and the separable closure of $E$ by, respectively, $E_{\text {alg }}$ and $E_{\text {sep. }}$. If $L$ is Galois over $E$, we denote the Galois group by $\operatorname{Gal}(L / E)$. We let $G(E)=\operatorname{Gal}\left(E_{\text {sep }} / E\right)$ and define $\chi(E)=H^{1}(G(E), \mathbb{Q} / \mathbb{Z})$, where $\mathbb{Q} / \mathbb{Z}$, the group of rationals mod 1 , has the discrete topology and trivial $G(E)$ action. $\chi(E)$ is thus the group of continuous homomorphisms from $G(E)$ into $\mathbb{Q} / \mathbb{Z}$. We refer to the elements of $\chi(E)$ as characters. If $f \in \chi(E)$, then $f(G(E))$ is a finite subgroup of $\mathbb{Q} / \mathbb{Z}$, and hence cyclic. Thus the fixed field of the kernel of $f$ is a cyclic Galois extension $L$ of $E$ of degree equal to the order of $f$. We say that $f$ defines the cyclic extension $L / E$. Viewed as an element of $\operatorname{Hom}(\operatorname{Gal}(L / E), \mathbb{Q} / \mathbb{Z}), f$ uniquely

determines a generator, $\sigma$, of $\operatorname{Gal}(L / E)$ by the condition $f(\sigma)=\frac{1}{[L: E]}+\mathbb{Z}$. We refer to $\sigma$ as the generator of $\operatorname{Gal}(L / E)$ determined by $f$. Finally, suppose that $t$ 
is transcendental over $E$ and $f \in \chi(E)$ defines $L / E$. We sometimes identify $f$ as an element of $\chi(E(t))$ defining the cyclic extension $L(t) / E(t)$.

Let $\alpha \in \operatorname{Br}(E)$. The order of $\alpha \in \operatorname{Br}(E)$ is called the exponent of $\alpha$ and denoted $\exp (\alpha)$. The homomorphism from $\operatorname{Br}(E)$ to $\operatorname{Br}(L)$ induced by the map $A \rightarrow A \otimes_{E} L$ on central simple $E$-algebras will be called the restriction homomorphism and be denoted by $\operatorname{res}_{L / E}$. If $L$ is a finite separable extension of $E$, we denote the corestriction homomorphism from $\operatorname{Br}(L)$ to $\operatorname{Br}(E)$ by $\operatorname{cor}_{L / E}$. If $b \in E^{*}=E-\{0\}$ and $f \in \chi(E)$, we let $\Delta(f, b)$ denote the element of $\operatorname{Br}(E)$ corresponding to the cyclic crossed product $(L / E, \sigma, b)$, where $f$ defines the cyclic extension $L / E$ and $\sigma$ is the generator of $\operatorname{Gal}(L / E)$ determined by $f$. If $f$ defines a quadratic extension $E(\sqrt{c}) / E$ and $b \in E-\{0\}$, then $\Delta(f, b)$ is denoted by $(c, b)_{E}$; it is the class in the Brauer group of $E$ containing the quaternion $E$-algebra generated by $x$ and $y$ subject to the relations $x^{2}=c, y^{2}=b$, and $x y=-y x$.

All valuations will be assumed to be exponential, i.e. additively written nonarchimedean with values in $\mathbb{R} \cup \infty$. Suppose that $P$ is a place of $L$ and $\delta$ is the associated valuation. We denote the valuation ring of $\delta$ by both $\mathcal{O}_{P}$ and $\mathcal{O}_{\delta}$, the maximal ideal of $\mathcal{O}_{\delta}$ by both $\mathcal{M}_{P}$ and $\mathcal{M}_{\delta}$, the residue field $\mathcal{O}_{\delta} / \mathcal{M}_{\delta}$ by both $\bar{L}_{P}$ and $\bar{L}_{\delta}$, and the completion of $L$ at $\delta$ by both $L_{P}$ and $L_{\delta}$. If $l \in \mathcal{O}_{\pi}$, we denote the image of $l$ under the natural map $\mathcal{O}_{\pi} \rightarrow \mathcal{O}_{\pi} / \mathcal{M}_{\pi}$ by $\bar{l}$; similarly, if $f \in \mathcal{O}_{\pi}[t], \bar{f}$ denotes the image of $f$ in $\bar{L}_{\pi}[t]$. The restriction of $P$ (respectively, $\delta$ ) to $E$ will be denoted by $P_{E}$ (respectively, $\delta_{E}$ ); when no misunderstanding is likely, we sometimes write $P$ instead of $P_{E}$ and $\delta$ instead of $\delta_{E}$. If $P$ is trivial on $E$, we say that $P$ is a place of $L / E$. If $\delta: L \rightarrow \mathbb{Z}$ is discrete and $b \in \mathcal{M}_{\delta}$ with $\delta(b)=1$, we call $b$ a uniformizer for $P$ (and also for $\delta$ ).

We next discuss the specialization of Brauer classes. Suppose that $P$ is a place of $E$ whose associated valuation is discrete and whose residue field, $\bar{E}_{P}$, is perfect. Let $\operatorname{Br}\left(\mathcal{O}_{P}\right)$ denote the Brauer group of $\mathcal{O}_{P}$. By [Sa, Lemma 1.2], the homomorphism from $\operatorname{Br}\left(\mathcal{O}_{P}\right)$ to $\operatorname{Br}(E)$ induced by $\mathcal{O}_{P} \rightarrow E$ is an injection and allows us to view $\operatorname{Br}\left(O_{P}\right)$ as a subgroup of $\operatorname{Br}(E)$. Let $\rho_{P}$ denote the homomorphism from $\operatorname{Br}\left(\mathcal{O}_{P}\right)$ to $\operatorname{Br}\left(\mathcal{O}_{P} / \mathcal{M}_{P}\right)=\operatorname{Br}\left(\bar{E}_{P}\right)$ induced by the map $\mathcal{O}_{P} \rightarrow \mathcal{O}_{P} / \mathcal{M}_{P}$. We say that $\rho_{P}$ is defined on $\alpha \in \operatorname{Br}(E)$ if $\alpha \in \operatorname{Br}\left(\mathcal{O}_{P}\right)$. If $\rho_{P}$ is defined on $\alpha$, we call $\rho_{P}(\alpha)$ the specialization of $\alpha$ at the place $P$ of $E$. Now suppose that $L$ is a finite separable extension of $E, \rho_{P}$ is defined on $\alpha$, and $Q$ is a place of $E$ extending $P$. Since the composition $\mathcal{O}_{P} \rightarrow \mathcal{O}_{Q} \rightarrow \bar{L}_{\mathbb{Q}}$ equals the composition $\mathcal{O}_{P} \rightarrow \bar{E}_{P} \rightarrow \bar{L}_{Q}, \rho_{Q}$ is defined on $\operatorname{res}_{L / E}(\alpha)$ and $\rho_{Q}\left(\operatorname{res}_{L / E}(\alpha)\right)=\operatorname{res}_{L_{Q} / E_{P}}\left(\rho_{P}(\alpha)\right)$.

In what follows, we will be concerned with specializations of Brauer classes coming from rational function fields. Let $F(t)$ be the rational function field in one variable over a field $F$ of characteristic 0 and let $c \in F_{\text {alg. }}$. The place of $F(t) / F$ (i.e., trivial on $F$ ) defined by $t \rightarrow c$ will be denoted by $P_{c} ; P_{c}: F(t) \rightarrow F(c) \cup\{\infty\}$. We let $\mathcal{O}_{c}$ denote the valuation ring of $P_{c}$ and $\nu_{c}: F(t) \rightarrow \mathbb{Z}$ the associated normalized discrete valuation of $F(t)$. Let $\operatorname{Irr}(c, F)$ denote the monic irreducible polynomial in $F[t]$ having $c$ as a root. Then $\mathcal{O}_{c}$ is the localization of $F[t]$ at the prime ideal $(\operatorname{Irr}(c, F)) \subset F[t]$, and $\operatorname{Irr}(c, F)$ is a uniformizer for $\nu_{c}$. We denote $\rho_{P_{c}}$ by $\rho_{c} ; \rho_{c}: \operatorname{Br}\left(\mathcal{O}_{c}\right) \rightarrow \operatorname{Br}(F(c))$.

Let $F$ be a field of characteristic 0 and let $F(t)$ be the rational function field in one variable over $F$. The Auslander-Brumer-Faddeev theorem provides an explicit description of the elements of $\operatorname{Br}(F(t))$; we next review this result. The version below appears in [FS1, p. 51]. 
The Auslander-Brumer-Faddeev Theorem. Let $F$ be a field of characteristic 0 and let $F(t)$ be the rational function field in one variable over $F$. Let $\alpha \in$ $\operatorname{Br}(F(t))$. Then there exist $\alpha_{0} \in \operatorname{Br}(F)$, a finite set $\left\{a_{1}, \ldots, a_{w}\right\} \subset F_{\text {alg }}$ such that $\operatorname{Irr}\left(a_{i}, F\right) \neq \operatorname{Irr}\left(a_{j}, F\right)$ for $i \neq j$, and a corresponding set of characters $f_{i} \in \chi\left(F\left(a_{i}\right)\right)$ for $i=1, \ldots, w$, such that

$$
\alpha=\operatorname{res}_{F(t) / F}\left(\alpha_{0}\right)+\sum_{i=1}^{w} \operatorname{cor}_{F\left(a_{i}, t\right) / F(t)}\left(\Delta\left(f_{i}, t-a_{i}\right)\right),
$$

where $f_{i} \in \chi\left(F\left(a_{i}\right)\right)$ is viewed as an element of $\chi\left(F\left(a_{i}, t\right)\right)$ for $i=1, \ldots, w$. This decomposition is unique if each $a_{i}$ is required to be an element of some fixed set containing exactly one root for each monic irreducible polynomial in $F[t]$. Moreover, $\exp (\alpha)$ equals the least common multiple of $\exp \left(\alpha_{0}\right)$ and the orders of the characters $f_{i}$ for $i=1, \ldots, w$.

We refer to $\alpha_{0}$ as the constant component of $\alpha$, and we will call the various classes $\operatorname{cor}_{F\left(a_{i}, t\right) / F(t)}\left(\Delta\left(f_{i}, t-a_{i}\right)\right)$ the basic components of $\alpha$.

Let $\alpha \in \operatorname{Br}(F(t))$ be as described in the statement of the Auslander-BrumerFaddeev theorem, and let $c \in F$. Then $\rho_{c}(\alpha)$, the specialization of $\alpha$ under the place $P_{c}$ of $F(t) / F$, is defined if and only if $c \neq a_{i}$ for $i=1, \ldots, w$. If this is the case, then $\rho_{c}(\alpha)=\alpha_{0}+\sum_{i=1}^{w} \operatorname{cor}_{F\left(a_{i}\right) / F}\left(\Delta\left(f_{i}, c-a_{i}\right)\right)$ [FSS, Lemma 2.2].

Let $c$ and $\alpha$ be as above, let $K$ be a finite extension of $F(t)$, let $\beta=\operatorname{res}_{K / F(t)}(\alpha)$, and let $Q$ be a place of $K$ extending the place $P_{c}$ of $F(t) / F$. Since $\rho_{c}\left(\operatorname{res}_{K / F(t)}(\alpha)\right)=$ $\operatorname{res}_{\bar{K}_{Q} / \overline{F(t)}_{P}}\left(\rho_{c}(\alpha)\right)$, it follows that

$$
\rho_{Q}(\beta)=\operatorname{res}_{K_{Q} / F}\left(\alpha_{0}+\sum_{i=1}^{w} \operatorname{cor}_{F\left(a_{i}\right) / F}\left(\Delta\left(f_{i}, c-a_{i}\right)\right)\right) .
$$

Let $E$ be a field, let $\alpha \in \operatorname{Br}(E)$, and suppose that $E$ has a discrete valuation $\pi$ such that the characteristic of $\bar{E}_{\pi}$ does not divide $\exp (\alpha)$. In what follows we will prove, for certain $\alpha$, that $\alpha \neq 0$ by using the character map $\chi_{\pi}: \operatorname{Br}(E) \rightarrow \chi\left(\bar{E}_{\pi}\right)$. We next review this map.

Fix a uniformizer $b \in E_{\pi}$. By a theorem of Witt [Se1, Ch. XII], every element $\alpha$ of $\operatorname{Br}\left(E_{\pi}\right)$ can be uniquely expressed in the form $\alpha=\beta+\Delta(f, b)$, where the character $f$ defines a cyclic unramified extension of $E_{\pi}$ and where $\beta$ is the inertial lift of a uniquely defined element $\beta_{0}$ of $\operatorname{Br}\left(\bar{E}_{\pi}\right)$. If $b^{\prime}$ is a different uniformizer, then $b=u b^{\prime}$, where $u \in E_{\pi}$ is a unit. Thus $\alpha=\beta+\Delta(f, b)=(\beta+\Delta(f, u))+\Delta\left(f, b^{\prime}\right)$, where now $\beta+\Delta(f, u)$ is the inertial lift of an element of $\operatorname{Br}\left(\bar{E}_{\pi}\right)$. Thus $f$ is uniquely determined by $\alpha$ and is independent of the choice of uniformizer. Since $f$ defines a cyclic unramified extension of $E_{\pi}$, we may view $f$ as an element of $\chi\left(\bar{E}_{\pi}\right)$. We define the character map, $\chi: \operatorname{Br}\left(E_{\pi}\right) \rightarrow \chi\left(\bar{E}_{\pi}\right)$, by $\alpha \rightarrow f$, and we define the character map at $\pi, \chi_{\pi}: \operatorname{Br}(E) \rightarrow \chi\left(\bar{E}_{\pi}\right)$ to be the composition $\chi \circ \operatorname{res}_{E_{\pi} / E}$.

We conclude this section with a technical result which is needed for the proof of our main theorem. Suppose that $K$ is a finite separable extension of a field $E$ and $\pi$ is a valuation of $E$. If there exists a primitive element $b$ for $K$ over $E$ such that $\operatorname{Irr}(b, E) \in \mathcal{O}_{\pi}[x]$ and $\operatorname{Irr}(b, E)$ has distinct roots modulo $\mathcal{M}_{\pi}$, then $\pi$ is unramified in $K$ (e.g., [We, Theorem 3-2-6] for the discrete case). While this is an important and frequently used criterion for recognizing that $\pi$ is unramified in $K$, it is possible for $\pi$ to be unramified in $K$ without such a primitive element $b$ existing. For example, suppose that $\bar{E}_{\pi}$ is finite, $\pi$ splits completely in $K$, and $[K: E]>\left|\bar{E}_{\pi}\right|$. If $b \in K$ is any primitive element for $K$ over $E$ such that $\operatorname{Irr}(b, E) \in \mathcal{O}_{\pi}[x]$, then 
$\operatorname{Irr}(b, E)$ splits completely modulo $\mathcal{M}_{\pi}$. But $\operatorname{Irr}(b, E)$ clearly has multiple roots modulo $\mathcal{M}_{\pi}$ since its degree is larger than the number of elements in $\mathcal{O}_{\pi} / \mathcal{M}_{\pi}$. The result we need asserts that if $\bar{E}_{\pi}$ is infinite and $\pi$ is unramified in $K$, then there exist $b$ with this property. Since it may be of independent interest, we prove that if $\bar{E}_{\pi}$ is infinite, then $\pi$ is unramified in $K$ if and only if such $b$ exist.

Proposition 2.1. Assume that $\pi$ is a valuation of a field $E$ such that the residue field $\bar{E}_{\pi}$ is infinite. Let $\mathcal{O}_{\pi}$ be the valuation ring of $\pi$, let $\mathcal{M}_{\pi}$ be the maximal ideal of $\mathcal{O}_{\pi}$, and let $K$ be a finite separable extension of $E$. Then $\pi$ is unramified in $K$ if and only if there exists a primitive element $b$ for $K$ over $E$ such that $\operatorname{Irr}(b, E) \in \mathcal{O}_{\pi}[t]$ and $\operatorname{Irr}(b, E)$ is separable modulo $\mathcal{M}_{\pi}$.

Proof. Let $\left\{\delta_{1}, \ldots, \delta_{r}\right\}$ be the complete set of inequivalent extensions of $\pi$ to $K$. We identify $E_{\pi}$ with the closure of $E$ in the completion $K_{\delta_{j}}$.

Suppose first that $\pi$ is unramified in $K$. Then for each $j, \bar{K}_{\delta_{j}}$ is separable over $\bar{E}_{\pi}$. For each $j=1, \ldots, r$, pick $k_{j} \in \mathcal{O}_{\delta_{j}} \subset K$ such that $\bar{k}_{j}$ is a primitive element for $\bar{K}_{\delta_{j}}$ over $\bar{E}_{\pi}$, and let $h_{j}(t)=\operatorname{Irr}\left(\bar{k}_{j}, \bar{E}_{\pi}\right)$. Since $\bar{E}_{\pi}$ is infinite, we may assume that the $k_{j}$ have been chosen so that the $h_{j}(t)$ are all different. By the weak approximation theorem [We Theorem 1-2-3], there exists $b \in K$ such that $\delta_{j}\left(b-k_{j}\right)>0$ for all $j=1, \ldots, r$. We claim that $b$ has the desired properties.

Let $f(t)=\operatorname{Irr}(b, E)$. Since $k_{j} \in \mathcal{O}_{\delta_{j}}$ and $\delta_{j}\left(b-k_{j}\right)>0, b$ is in $\mathcal{O}_{\delta_{j}}$ for each $j=1, \ldots, r$. Thus $f(t) \in \mathcal{O}_{\pi}[t]$, since the roots (and hence the coefficients) of $f(t)$ are integral over $\mathcal{O}_{\pi}$. Fix $j$, and let $\bar{b}_{j}$ be the image of $b$ in $\bar{K}_{\delta_{j}}$. Since $\delta_{j}\left(b-k_{j}\right)>0$, we have $\bar{b}_{j}=\bar{k}_{j}$, and so $\operatorname{Irr}\left(\bar{b}_{j}, \bar{E}_{\pi}\right)=h_{j}(t)$. Since $f(b)=0$, we have $\bar{f}\left(\bar{b}_{j}\right)=0$, where $\bar{f}(t)$ is the image of $f(t)$ in $\bar{E}_{\pi}[t]$. It follows that $h_{j}(t)$ divides $\bar{f}(t)$ in $\bar{E}_{\pi}[t]$. Since this holds for all $j=1, \ldots, r$, and since the $h_{j}(t)$ are pairwise relatively prime, $h_{1}(t) \cdots h_{r}(t)$ divides $\bar{f}(t)$ in $\bar{E}_{\pi}[t]$. Since $\pi$ is unramified in $K$,

$$
\begin{aligned}
\sum_{j=1}^{r} \operatorname{deg}\left(h_{j}\right) & \leq \operatorname{deg}(\bar{f}(t))=\operatorname{deg}(f(t))=[E(b): E] \leq[K: E] \\
& =\sum_{j=1}^{r}\left[\bar{K}_{\delta_{j}}: \bar{E}_{\pi}\right]=\sum_{j=1}^{r}\left[\bar{E}_{\pi}\left(\bar{k}_{j}\right): \bar{E}_{\pi}\right]=\sum_{j=1}^{r} \operatorname{deg}\left(h_{j}(t)\right) .
\end{aligned}
$$

We conclude that equality must hold throughout, and so $K=E(b)$ and $\bar{f}(t)=$ $h_{1}(t) \cdots h_{r}(t)$. Since each $h_{i}(t)$ is separable and the $h_{i}(t)$ are distinct, $\bar{f}(t)$ is separable and so $b$ has the desired properties.

Conversely, suppose that there exists a primitive element $b$ for $K / E$ such that $f(t)=\operatorname{Irr}(b, E) \in \mathcal{O}_{\pi}[t]$ and $\bar{f}(t)$ is separable. Since $\pi$ has $r$ inequivalent extensions to $K, f(t)$ factors into a product of $r$ distinct monic irreducible polynomials in $E_{\pi}[t]$. Suppose that $f(t)=g_{1}(t) \cdots g_{r}(t)$ is this factorization. Let $\widetilde{\mathcal{O}}_{\pi} \subset E_{\pi}$ be the completion of $\mathcal{O}_{\pi}$. Then each $g_{j}(t) \in \widetilde{\mathcal{O}}_{\pi}[t]$, since the roots (and hence the coefficients) of $g_{j}(t)$ are integral over $\widetilde{\mathcal{O}}_{\pi}$. By Hensel's lemma [We, Corollary 2-2-5], for each $j=1, \ldots, r$ there exist a monic irreducible polynomial $w_{j}(t) \in \bar{E}_{\pi}[t]$ and a positive integer $m_{j}$ such that $\bar{g}_{j}(t)=w_{j}(t)^{m_{j}}$. But then $\bar{f}(t)=\bar{g}_{1}(t) \cdots \bar{g}_{r}(t)=$ $w_{1}(t)^{m_{1}} \cdots w_{r}(t)^{m_{r}}$. Since $\bar{f}(t)$ is separable by assumption, each $m_{j}=1$, and so each $\bar{g}_{j}(t)$ is irreducible in $\bar{E}_{\pi}[t]$.

Let $\Omega$ denote a fixed algebraic closure of $E_{\pi}$, let $\pi$ denote the extension of $\pi$ to $\Omega$, and let $b_{j} \in \Omega$ be a root of $g_{j}(t)$. We may assume the $\delta_{j}$ have been numbered 
so that $\delta_{j}$ is defined by $\delta_{j}(b)=\pi\left(b_{j}\right)$. Then $K_{\delta_{j}}=E_{\pi}\left(b_{j}\right)$. Since $g_{j}\left(b_{j}\right)=0$, $\bar{g}_{j}\left(\bar{b}_{j}\right)=0$. It follows that

$$
\begin{aligned}
{[K: E] } & =[E(b): E]=\operatorname{deg}(f(t))=\sum_{j=1}^{r} \operatorname{deg}\left(g_{j}(t)\right)=\sum_{j=1}^{r} \operatorname{deg}\left(\bar{g}_{j}(t)\right) \\
& =\left[\bar{E}_{\pi}\left(\bar{b}_{j}\right): \bar{E}_{\pi}\right] \leq \sum_{j=1}^{r}\left[\bar{K}_{\delta_{j}}: \bar{E}_{\pi}\right] \leq \sum_{j=1}^{r}\left[K_{\delta_{j}}: E_{\pi}\right]=[K: E] .
\end{aligned}
$$

We conclude that equality must hold throughout, and so $\pi$ is unramified in $K$.

Remark 2.1. Although we have restricted our attention to real valuations, the above proof shows that Proposition 2.1 is valid more generally if $\pi$ is a Krull valuation. In the first part of the above argument, in place of the weak approximation theorem for real valuations one uses the approximation theorem for Krull valuations [E] Theorem 11.16]. In the converse direction, one replaces $E_{\pi}$ by the Henselization of $E$ with respect to $\pi$ and uses Hensel's condition [E, Corollary 16.6] in place of Hensel's lemma to conclude that $\bar{g}_{j}(t)=w_{j}(t)^{m_{j}}$. We thank the referee for simplifying our original proof of Proposition 2.1 and for pointing out the validity of Proposition 2.1 for general Krull valuations.

We will need the existence of a suitable primitive element $b$ as above when we are given a finite set of valuations of $E$ each of which is unramified in $K$. We conclude this section with this result.

Corollary 2.2. Assume that $\pi_{1}, \ldots, \pi_{u}$ are a finite set of valuations of a field $E$ such that each residue field $\bar{E}_{\pi_{i}}$ is infinite. For each $i$, let $\mathcal{O}_{\pi_{i}}$ be the valuation ring of $\pi_{i}$ and $\mathcal{M}_{\pi_{i}}$ the maximal ideal of $\mathcal{O}_{\pi_{i}}$. Let $K$ be a finite separable extension of $E$ in which each $\pi_{i}$ is unramified. Then there exists a primitive element $b$ for $K$ over $E$ such that, for each $i=1, \ldots, r, \operatorname{Irr}(b, E) \in \mathcal{O}_{\pi_{i}}[t]$ and $\operatorname{Irr}(b, E)$ is separable modulo $\mathcal{M}_{\pi_{i}}$.

Proof. Let $\left\{\delta_{i, 1}, \ldots, \delta_{i, r(i)}\right\}$ be the complete set of inequivalent extensions of $\pi_{i}$ to $K$. For each pair $(i, j)$ with $1 \leq i \leq u$ and $1 \leq j \leq r(i)$, pick $k_{i, j} \in K$ such that $k_{i, j}$ is in the valuation ring of $\delta_{i, j}$ and such that, if $\bar{k}_{i, j}$ denotes the image of $k_{i, j}$ in the residue field of $K$ at $\delta_{i, j}$, then $\bar{k}_{i, j}$ is a primitive element for that residue field over $\bar{E}_{\pi_{i}}$. Moreover, since each $\bar{E}_{\pi_{i}}$ is infinite, we may assume that the $k_{i, j}$ have been chosen so that $\operatorname{Irr}\left(\bar{k}_{i, j}, \bar{E}_{\pi}\right) \neq \operatorname{Irr}\left(\bar{k}_{i, l}, \bar{E}_{\pi}\right)$ for each $i$ and each $j \neq l$. By the weak approximation theorem there exists $b \in K$ such that $\delta_{i, j}\left(b-k_{i, j}\right)>0$ for all $i, j$. The proof of Proposition 2.1 shows that $b$ is a primitive element for $K$ over $E$ such that, for each $i, \operatorname{Irr}(b, E) \in \mathcal{O}_{\pi_{i}}[t]$ and $\operatorname{Irr}(b, E)$ is separable modulo $\mathcal{M}_{\pi_{i}}$.

\section{The Main Theorem}

In this section we prove our main result:

Theorem 3.1. Let $F$ be either a number field or a field finitely generated of transcendence degree $\geq 1$ over a Hilbertian field of characteristic 0 , let $F(t)$ be the rational function field in one variable over $F$, and let $K$ be a finite extension of $F(t)$. Let $\alpha \in \operatorname{Br}(F(t))$ and suppose that

$$
\alpha=\operatorname{res}_{F(t) / F}\left(\alpha_{0}\right)+\sum_{i=1}^{w} \operatorname{cor}_{F\left(a_{i}, t\right) / F(t)}\left(\Delta\left(f_{i}, t-a_{i}\right)\right),
$$


where $\alpha_{0} \in \operatorname{Br}(F)$ is the constant component of $\alpha, f_{i} \in \chi\left(F\left(a_{i}\right)\right)$ is viewed as an element of $\chi\left(F\left(a_{i}, t\right)\right)$ for $i=1, \ldots, w$, and $\operatorname{Irr}\left(a_{i}, F\right) \neq \operatorname{Irr}\left(a_{j}, F\right)$ for $i \neq j$. Let $P_{i}=P_{a_{i}}$ be the place of $F(t) / F$ defined by $t \rightarrow a_{i}$. Assume that $P_{i}$ is unramified in $K$ for $1 \leq i \leq u$, and let $Q_{i}$ be an extension of $P_{i}$ to $K$ for $1 \leq i \leq u$. Let $\beta=\operatorname{res}_{K / F(t)}(\alpha)$, and let $n$ be the least common multiple of the orders of the characters $\left\{\operatorname{res}_{K_{Q_{i}} / F\left(a_{i}\right)}\left(f_{i}\right) \mid i=1 \ldots, u\right\}$. Then there exist infinitely many $c \in F$ such that $\rho_{c}$ is defined on $\alpha$ and such that the place $P_{c}$ of $F(t) / F$ has an extension to a place $T$ of $K / F$ such that $n$ divides the exponent of the specialization, $\rho_{T}(\beta) \in \operatorname{Br}\left(\bar{K}_{T}\right)$, of $\beta$. In particular, $n$ divides $\exp (\beta)$.

Remark 3.1. To clarify the content of Theorem 3.1, consider the case when $\alpha$ is a single basic component, $\alpha=\operatorname{cor}_{F(b, t) / F(t)}(\Delta(f, t-b))$. $\alpha$ is determined by the two pieces of data, $b \in F_{\text {alg }}$ and $f \in \chi(F(b))$. Since $\beta=\operatorname{res}_{K / F(t)}(\alpha)$, the specialization properties of $\beta$ are determined by $b$ and $f$. It is natural, then, to consider the behavior in $K$ of the place $P_{b}$ of $F(t) / F$ defined by $t \rightarrow b$. As we shall see in Example 2 of Section 4, Theorem 3.1 does not hold if $P_{b}$ ramifies in $K$. Assume, then, that $P_{b}$ is unramified in $K$, let $Q$ be an extension of $P_{b}$ to $K$, and suppose that $f$ defines the cyclic extension $L / F(b)$. Note that $\bar{K}_{Q} \supseteq \overline{F(t)}_{P_{b}}=F(b)$. Let $n=\left[L \bar{K}_{Q}: \bar{K}_{Q}\right]$, so $n$ is the order of $\operatorname{res}_{\bar{K}_{Q} / F(b)}(f)$. Then Theorem 3.1 asserts that there exist infinitely many $c \in F$ such that $\rho_{c}$ is defined on $\alpha$ and such that the place $P_{c}$ of $F(t) / F$ has an extension to a place $T$ of $K / F$ such that $n$ divides the exponent of the specialization, $\rho_{T}(\beta) \in \operatorname{Br}\left(\bar{K}_{T}\right)$, of $\beta$. In particular, if $[L: F(b)]=\left[L \bar{K}_{Q}: \bar{K}_{Q}\right]$, then since neither restriction nor specialization can increase exponent, $\exp (\alpha)=\exp (\beta)=\exp \left(\rho_{c}(\beta)\right)$. It should now be clear how Theorem 3.1 gives sufficient conditions on $K$ and $\alpha \in \operatorname{Br}(F(t))$ so that there exist infinitely many $c \in F$ such that $\rho_{c}$ is defined on $\alpha$ and such that $P_{c}$ can be extended to a place $T$ of $K / F$ which preserves the exponent of $\beta=\operatorname{res}_{K / F(t)}(\alpha)$.

Before proceeding to the proof of Theorem 3.1, we recall a notion introduced in [FSS, p. 925]. Let $F$ be as in the statement of Theorem 3.1, let $E$ be a finite algebraic extension of $F$, and let $L$ be a finite cyclic extension of $E$. A valuation $\pi$ of $L$ is said to be good for the tower $F \subseteq E \subseteq L$ if $E_{\pi}=F_{\pi}$ and $\left[L_{\pi}: E_{\pi}\right]=[L: E]$. (Recall our convention that if $\pi$ is a valuation of a field $E$ and $M$ is a subfield of $E$, then we also use $\pi$ to denote the restriction, $\pi_{M}$, of $\pi$ to $M$.) For the fields $F$ we are considering, it is shown in [FSS, p. 927] that for any tower $F \subseteq E \subseteq L$ as above, there exist infinitely many discrete valuations of $L$ which are good for that tower. (There is a small gap in the proof of this result given in [FSS]; a corrected proof appears in [FS3, p. 534].) If $F$ is a finite extension of $F_{0}(u)$, where $F_{0}$ is a Hilbertian field of characteristic 0 and $u$ is transcendental over $F_{0}$, we will only consider valuations of $F$ that are trivial on $F_{0}$; in particular, the residue fields under these valuations will have characteristic 0 .

Proof of Theorem 3.1. Let the context be as in the statement of Theorem 3.1 and let $\mathcal{O}_{i}=\mathcal{O}_{P_{i}}$ be the valuation ring of $P_{i}$ in $F(t)$ for $i=1 \ldots, u$. Then $\mathcal{O}_{i}$ modulo its maximal ideal equals $F\left(a_{i}\right)$. For $z \in K$, let $\operatorname{Irr}(z, F(t))$ denote the monic irreducible polynomial in $F(t)[x]$ having $z$ as a root. By Corollary 2.2, there exists a primitive element $b$ for $K$ over $F(t)$ such that, for $i=1, \ldots, u, \operatorname{Irr}(b, F(t)) \in$ $\mathcal{O}_{i}[x]$ and $\operatorname{Irr}(b, F(t))$ is separable in $F\left(a_{i}\right)[x]$. Let $g(t, x)=\operatorname{Irr}(b, F(t))$. Then the discriminant, $\operatorname{disc}\left(g\left(a_{i}, x\right)\right)$, of $g\left(a_{i}, x\right)$ is non-0 for $i=1, \ldots, u$. 
Let $i \in\{1, \ldots, u\}$ be arbitrary but fixed. Since $b$ is integral over $\mathcal{O}_{i}, Q_{i}$ is finite on $b$. Let $b_{i}=Q_{i}(b)$. Then $b_{i}$ is the image of $b$ in the residue field, $\bar{K}_{Q_{i}}$, of $K$ under the place $Q_{i}$. Since $g(t, x) \in \mathcal{O}_{i}[x]$ and $g(t, b)=0, g\left(a_{i}, b_{i}\right)=Q_{i}(g(t, b))=0$. Since $P_{i}$ is unramified in $K$ by assumption and $g\left(a_{i}, x\right)$ is separable in $F\left(a_{i}\right)[x]$, $\bar{K}_{Q_{i}}=F\left(a_{i}, b_{i}\right)$.

Let $f_{i} \in \chi\left(F\left(a_{i}\right)\right)$ define the cyclic extension $L_{i} / F\left(a_{i}\right)$. Then $\operatorname{res}_{\bar{K}_{Q_{i}} / F\left(a_{i}\right)}\left(f_{i}\right)$ defines the cyclic extension $L_{i}\left(b_{i}\right) / F\left(a_{i}, b_{i}\right)$. As noted immediately after the statement of Theorem 3.1, the hypotheses on $F$ imply that there are infinitely many discrete valuations $\pi_{i}$ of $L_{i}\left(b_{i}\right)$ which are good for the tower $F \subseteq F\left(a_{i}, b_{i}\right) \subseteq L_{i}\left(b_{i}\right)$; that is, $F\left(a_{i}, b_{i}\right)_{\pi_{i}}=F_{\pi_{i}}$ and $\left[L_{i}\left(b_{i}\right)_{\pi_{i}}: F\left(a_{i}, b_{i}\right)_{\pi_{i}}\right]=\left[L_{i}\left(b_{i}\right): F\left(a_{i}, b_{i}\right)\right]$. We will require that $\pi_{i}$ satisfy several additional conditions.

Since $F$ either is a number field or is finitely generated of transcendence degree $\geq 1$ over a Hilbertian field of characteristic 0 , it is clear that, in addition to requiring that $\pi_{i}$ be good for the tower $F \subseteq F\left(a_{i}, b_{i}\right) \subseteq L_{i}\left(b_{i}\right)$, we can also require that the characteristic of $\bar{F}_{\pi_{i}}$ does not divide $\exp (\alpha)$. Next, for $\pi$ a discrete valuation of $F$, let $\chi_{\pi}$ denote the character map $\operatorname{Br}(F) \rightarrow \operatorname{Br}\left(F_{\pi}\right) \rightarrow \chi\left(\bar{F}_{\pi}\right)$ introduced in section 2 . Then there are only finitely many $\pi$ such that $\chi_{\pi}\left(\alpha_{0}\right) \neq 0$, where $\alpha_{0}$ is the constant component of $\alpha$. (This can be seen, for example, by choosing an $F$-basis for $\alpha_{0}$ and noting that $\operatorname{res}_{F_{\pi} / F}\left(\alpha_{0}\right)$ is an inertial algebra if the coefficients that define the multiplication in $\alpha_{0}$ using that basis are $\pi$-units.) We will require that $\chi_{\pi_{i}}\left(\alpha_{0}\right)=0$. Finally, let $E$ be a Galois extension of $F$ containing $a_{j}$ for all $1 \leq j \leq w$ and $L_{j}\left(b_{j}\right)$ for all $j=1, \ldots, u$.

By the remarks above, it should be clear that there exist infinitely many discrete valuations $\pi_{i}$ of $E$ such that:

(1) $\pi_{i}$ is good for the tower $F \subseteq F\left(a_{i}, b_{i}\right) \subseteq L_{i}\left(b_{i}\right)$, i.e., $F\left(a_{i}, b_{i}\right)_{\pi_{i}}=F_{\pi_{i}}$, and $\left[L_{i}\left(b_{i}\right)_{\pi_{i}}: F\left(a_{i}, b_{i}\right)_{\pi_{i}}\right]=\left[L_{i}\left(\bar{b}_{i}\right): F\left(a_{i}, b_{i}\right)\right]$

(2) $\pi_{i}$ is unramified in $E$;

(3) the characteristic of $\bar{F}_{\pi_{i}}$ does not divide $\exp (\alpha)$;

(4) $\chi_{\pi_{i}}\left(\alpha_{0}\right)=0$

(5) the following are $\pi_{i}$-units: the coefficients in $F$ of $g(t, x)$, all algebraic conjugates of $a_{j}$ for $1 \leq j \leq w$ and all non-zero differences of such conjugates, all algebraic conjugates of $b_{j}$ for $1 \leq j \leq u$, all differences $a_{i}-a_{j}$ for $i \neq j$, and $\operatorname{disc}\left(g\left(a_{i}, x\right)\right)$.

For each $i=1, \ldots, u$, choose $\pi_{i}$ satisfying the above conditions and, in addition, the requirement that:

(6) $\left(\pi_{i}\right)_{F}$ and $\left(\pi_{j}\right)_{F}$ are inequivalent if $i \neq j$.

Let $i \in\{1, \ldots, u\}$. By $(1), a_{i} \in F_{\pi_{i}}$. Let $a_{i}^{\prime} \in F$ be chosen so that $\pi_{i}\left(a_{i}-a_{i}^{\prime}\right) \geq 2$ and let $e_{i} \in F$ be a uniformizer for $\left(\pi_{i}\right)_{F}$. Our hypotheses on $F$ imply that $F$ is Hilbertian. We also know that $g(t, x)$ is irreducible in $F[t, x]$ and the set of primes $\pi_{i}, 1 \leq i \leq u$, satisfies the weak approximation theorem. It follows from $\mathrm{G}$ Lemma 3.4] that there exist infinitely many $c \in F$ satisfying the following:

(7) $g(c, x)$ is defined and irreducible in $F[x]$;

(8) for each $1 \leq i \leq u, \pi_{i}\left(c-\left(a_{i}^{\prime}+e_{i}\right)\right) \geq 2$.

Let $c \in F$ satisfy (7) and (8). The place $P_{c}$ of $F(t) / F$ can be extended to $K$ by sending $b$ to a root of $g(c, x)$. Since $g(c, x)$ is irreducible in $F[x], P_{c}$ is unramified in $K$ and any two extensions of $P_{c}$ to $K$ are equivalent. Let $d \in F_{\text {alg }}$ be a root of $g(c, x)$ and let $T$ be the place of $K / F$ extending $P_{c}$ defined by $b \rightarrow d$. Then the 
residue field, $\bar{K}_{T}$, of $T$ is $F(d)$. We will show that $T$ satisfies the conclusions of Theorem 3.1.

We first show:

(9) for each $i=1, \ldots, u, \pi_{i}$ is unramified in $F(d)$ and has an extension $\delta_{i}$ to $F(d)$ with $F(d)_{\delta_{i}}=F_{\pi_{i}}$.

Fix $i \in\{1, \ldots, u\}$. By (8), $c$ is a $\pi_{i}$-integer. By (5), every coefficient of $g(c, x)$ is a $\pi_{i}$-integer. Let $\overline{g(c, x)}$ be the image of $g(c, x)$ in $\bar{F}_{\pi_{i}}[x]$. By (5), (8), and (1), $\overline{g(c, x)}=\overline{g\left(a_{i}, x\right)} \in \overline{F\left(a_{i}\right)} \pi_{i}[x]=\bar{F}_{\pi_{i}}[x]$. Since $b_{i}$ is a $\pi_{i}$-unit, $\overline{F\left(a_{i}, b_{i}\right)_{\pi_{i}}}=\bar{F}_{\pi_{i}}$, and $g\left(a_{i}, b_{i}\right)=0$, it follows that the image $\bar{b}_{i}$ of $b_{i}$ in $\overline{F\left(a_{i}, b_{i}\right)} \bar{\pi}_{i}$ is a root in $\bar{F}_{\pi_{i}}$ of $\overline{g(c, x)}$. Since $\operatorname{disc}\left(g\left(a_{i}, x\right)\right)$ is a $\pi_{i}$-unit, $\overline{g(c, x)}$ is separable over $\bar{F}_{\pi_{i}}$. By Hensel's lemma, $g(c, x)$ has a root in $F_{\pi_{i}}$. Since $g(c, x)=\operatorname{Irr}(d, F)$, (9) follows.

Let $\chi_{\delta_{i}}$ denote the ramification map from $\operatorname{Br}(F(d))$ to $\chi\left(\overline{F(d))}_{\delta_{i}}=\chi\left(\bar{F}_{\pi_{i}}\right)\right.$ for $i=$ $1, \ldots, u$. To prove Theorem 3.1, it suffices to show that for each $i=1, \ldots, u$, the order of $\chi_{\delta_{i}}\left(\rho_{T}(\beta)\right)$ equals the order of $\operatorname{res}_{\bar{K}_{Q_{i}} / F\left(a_{i}\right)}\left(f_{i}\right)$. Recall that res $\bar{K}_{Q_{i}} / F\left(a_{i}\right)\left(f_{i}\right)$ defines the cyclic extension $L_{i}\left(b_{i}\right) / F\left(a_{i}, b_{i}\right)$. Let $M_{i}=L_{i} \cap F\left(a_{i}, b_{i}\right)$, so

$$
\left[L_{i}\left(b_{i}\right): F\left(a_{i}, b_{i}\right)\right]=\left[L_{i}: M_{i}\right] .
$$

Thus we must show that, for each $i=1, \ldots, u$, the order of $\chi_{\delta_{i}}\left(\rho_{T}(\beta)\right)$ equals $\left[L_{i}: M_{i}\right]$. Fix $i \in\{1, \ldots, u\}$.

We next show:

(10) Let $1 \leq j \leq w, j \neq i$. Then $c-a_{i}$ is a uniformizer in $F_{\pi_{i}}$ and $c-a_{j}$ is a $\pi_{i}$-unit in $F_{\pi_{i}}\left(a_{j}\right)$.

Write $c-a_{i}=\left(c-\left(a_{i}^{\prime}+e_{i}\right)\right)+\left(a_{i}^{\prime}-a_{i}\right)+e_{i}$. Recall that $e_{i} \in F$ is a uniformizer for $\pi_{i}$, and $a_{i}^{\prime} \in F$ was chosen so that $\pi_{i}\left(a_{i}-a_{i}^{\prime}\right) \geq 2$. It follows from (8) that $\pi_{i}\left(c-a_{i}\right)=1$, and so $c-a_{i}$ is a uniformizer for $\pi_{i}$. Next, for $j \neq i$, write $c-a_{j}=\left(c-a_{i}\right)+\left(a_{i}-a_{j}\right)$. Since $\pi_{i}\left(c-a_{i}\right)=1$ but $\pi_{i}\left(a_{i}-a_{j}\right)=0$ by (5), we have $\pi_{i}\left(c-a_{j}\right)=0$, proving (10).

From the commutative diagram

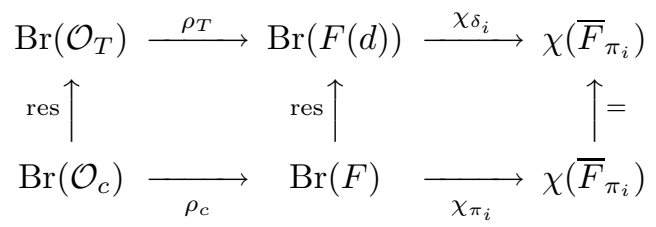

we see that it suffices to prove that the order of $\chi_{\pi_{i}}\left(\rho_{c}(\alpha)\right)$ equals $\left[L_{i}: M_{i}\right]$. By [FSS, Lemma 2.2],

$$
\rho_{c}(\alpha)=\alpha_{0}+\sum_{j=1}^{w} \operatorname{cor}_{F\left(a_{j}\right) / F}\left(\Delta\left(f_{j}, c-a_{j}\right)\right) .
$$

By $(4), \chi_{\pi_{i}}\left(\alpha_{0}\right)=0$, and by $(2)$ and $(10), \chi_{\pi_{i}}\left(\operatorname{cor}_{F\left(a_{j}\right) / F}\left(\Delta\left(f_{j}, c-a_{j}\right)\right)\right)=0$ if $j \neq i$. Thus

$$
\chi_{\pi_{i}}\left(\rho_{c}(\alpha)\right)=\chi_{\pi_{i}}\left(\operatorname{cor}_{F\left(a_{i}\right) / F}\left(\Delta\left(f_{i}, c-a_{i}\right)\right)\right) .
$$

For convenience, we denote $\pi_{i}$ by $\pi, a_{i}$ by $a, f_{i}$ by $f$, and so on. Let $\zeta_{1}$ be the restriction of $\pi$, viewed as a valuation of $E$, to $F(a)$. By (1), $F(a)_{\zeta_{1}}=F_{\pi}$. Let 
$\zeta_{1}, \ldots, \zeta_{m}$ be the inequivalent extensions of $\pi$ to $F(a)$. By [FSS, Theorem 1.4],

$$
\chi_{\pi}\left(\operatorname{cor}_{F(a) / F}(\Delta(f, c-a))\right)=\sum_{j=1}^{m} \operatorname{cor} \overline{F(a)}_{\zeta_{j}} / \bar{F}_{\pi}\left(\chi_{\zeta_{j}}(\Delta(f, c-a))\right) .
$$

Since there are $m$ inequivalent extensions of $\pi$ to $F(a), \operatorname{Irr}(a, F)$ factors as a product of $m$ irreducible polynomials in $F_{\pi}$. Let $a=d_{1}, d_{2}, \ldots, d_{m}$ be roots of these polynomials. Then $\zeta_{j}$ is defined by $\zeta_{j}(a)=d_{j}$. Since the $d_{j}$ are algebraic conjugates of $d_{1}=a=a_{i}, d_{1}-d_{j}$ is a $\pi$-unit for $j>1$ by (5). By (10), $c-d_{1}$ is a uniformizer for $F_{\pi}$. Since $c-d_{j}=\left(c-d_{1}\right)+\left(d_{1}-d_{j}\right), c-d_{j}$ is a $\pi$-unit for $j>1$. By definition of $\chi_{\zeta_{j}}, \chi_{\zeta_{j}}(\Delta(f, c-a))=0$ for $j>1$. For $j=1$ we have $F(a)_{\zeta_{1}}=F_{\pi}$, and so $\chi_{\pi}\left(\operatorname{cor}_{F(a) / F}(\Delta(f, c-a))=\chi_{\zeta_{1}}(\Delta(f, c-a))\right.$. Recall that $\chi_{\zeta_{1}}$ can be computed by going to the completion of $F(a)$ at $\zeta_{1}$. Consider $\operatorname{res}_{F(a)_{\zeta_{1}} / F(a)}(\Delta(f, c-a))=\Delta\left(\operatorname{res}_{F(a)_{\zeta_{1}} / F(a)}(f), c-d_{1}\right)$. Since $c-d_{1}$ is a uniformizer for $F(a)_{\zeta_{1}}$, the order of $\chi_{\zeta_{1}}(\Delta(f, c-a))$ equals the order of $\left.\operatorname{res}_{F(a)}\right)_{\zeta_{1}} / F(a)(f)$. But $f$ defines $L / F(a)$ and $\pi$ is good for the tower $F \subseteq F(a, b) \subseteq L(b)$. Thus $\pi$ is good for the tower $F \subseteq M \subseteq L$, and so $\operatorname{res}_{F(a)_{\zeta_{1}} / F(a)}(f)$ has order $[L: M]$, as was to be shown. This completes the proof of Theorem 3.1.

We note the following immediate corollary of Theorem 3.1, which will be referred to repeatedly in the final section of this paper.

Corollary 3.2. Let the context be as in Theorem 3.1 and suppose that $\alpha \in \operatorname{Br}(F(t))$ has prime exponent. Assume that there exists a basic component of $\alpha$ of the form $\operatorname{cor}_{F(a, t) / F(t)}(\Delta(f, t-a))$ such that the place $P_{a}$ of $F(t) / F$ sending $t \rightarrow a$ is unramified in $K$. Let $f$ define the cyclic extension $L / F(a)$. Assume that there exists a place $Q$ of $K / F$ extending $P_{a}$ such that $L \nsubseteq \bar{K}_{Q}$. Let $\beta=\operatorname{res}_{K / F(t)}(\alpha)$. Then $\exp (\beta)=\exp (\alpha)$, and there exist infinitely many $c \in F$ with $\rho_{c}$ defined on $\alpha$ and such that the place $P_{c}$ of $F(t) / F$ has an extension to a place $T$ of $K / F$ satisfying $\exp \left(\rho_{T}(\beta)\right)=\exp (\alpha)$.

\section{EXAMPLES}

In this section we will analyze several examples in the following simple special case. Let $\mathbb{Q}$ be the field of rational numbers, let $b \in \mathbb{Q}-\mathbb{Q}^{2}$, let $t$ be transcendental over $\mathbb{Q}$, and let $\alpha=(b, t)_{\mathbb{Q}(t)}$. Then $\alpha$ is a basic quaternionic Brauer class of exponent 2. Let $g(t)$ be a square-free polynomial in $\mathbb{Q}[t]$, let $K=\mathbb{Q}(t)(\sqrt{g(t)})$, and let $\beta=\operatorname{res}_{K / \mathbb{Q}(t)}(\alpha)$. In the context of Corollary 3.2, $a=0$ and $f$ defines the quadratic extension $\mathbb{Q}(\sqrt{b}) / \mathbb{Q}$. The place $P_{0}$ of $\mathbb{Q}(t) / \mathbb{Q}$ is unramified in $K$ if and only if $g(0) \neq 0$. Moreover, if this is the case and $Q$ is an extension of $P_{0}$ to $K$, then $\bar{K}_{Q}=\mathbb{Q}(\sqrt{g(0)})$. Thus, in our special situation, Corollary 3.2 becomes the following:

Corollary 3.2 (Special Case). Let $b \in \mathbb{Q}-\mathbb{Q}^{2}$, let $g(t)$ be a square-free polynomial in $\mathbb{Q}[t]$, let $\alpha=(b, t)_{\mathbb{Q}(t)}, K=\mathbb{Q}(t)(\sqrt{g(t)})$, and let $\beta=\operatorname{res}_{K / F(t)}(\alpha)$. Assume that $g(0) \neq 0$ and $\sqrt{b} \notin \mathbb{Q}(\sqrt{g(0)})$. Then $\exp (\beta)=2$, and there exist infinitely many $c \in \mathbb{Q}-\{0\}$ such that the place $P_{c}$ of $\mathbb{Q}(t) / \mathbb{Q}$ sending $t \rightarrow c$ has an extension to a place $T$ of $K / \mathbb{Q}$ with $\exp \left(\rho_{T}(\beta)\right)=2$.

We will present examples which show that the conclusions of Corollary 3.2 (Special Case) need not hold if either of the hypotheses $g(0) \neq 0$ or $\sqrt{b} \notin \mathbb{Q}(\sqrt{g(0)})$ 
is eliminated. We will also give an example showing that these hypotheses are not necessary for the conclusions of Corollary 3.2 (Special Case) to hold.

In what follows, we will make free use of the classification of Brauer classes over number fields by means of Hasse invariants; we refer the reader to $\mathrm{P}$, Chapter 18] for an exposition of this material. Since we will only be considering Brauer classes of exponent 1 or 2 , it turns out to be more convenient to use multiplicative notation for Hasse invariants. Suppose that $E$ is a number field, $A \in \operatorname{Br}(E)$ with $\exp (A) \leq 2$, and $\pi$ is a valuation of $E$. We define the multiplicative Hasse invariant of $A$ at $\pi, \operatorname{inv}_{\pi}(A)$, to be 1 if $\operatorname{res}_{E_{\pi} / E}(A)=0$ and to be -1 otherwise; in particular, $\operatorname{inv}_{\pi}(A)=-1$ corresponds to Hasse invariant $1 / 2$ in the usual additive notation. Now suppose that $a, b \in E-\{0\}$. We denote $\operatorname{inv}_{\pi}\left((a, b)_{E}\right)$ by $(a, b)_{\pi} ;$ if $E=\mathbb{Q}$ and $\pi$ is the $p$-adic valuation of $\mathbb{Q}$, we denote $(a, b)_{\pi}$ by $(a, b)_{p}$.

Now let $a, b \in \mathbb{Q}-\{0\}$ and let $p$ be a prime. Our notation for multiplicative Hasse invariants was chosen so as to be consistent with the usual notation for the norm residue symbol [We, page 249]. If $p$ is an odd prime and $a \in \mathbb{Q}$ is a $p$-unit, we let $\left(\frac{a}{p}\right)$ denote, as usual, the Legendre symbol. We will make frequent use of the following basic result [We, Proposition 6-6-4]: if $p$ is an odd prime and $u, v$ are units of $\mathbb{Q}_{p}$, then $\left(u p^{r}, v\right)_{p}=\left(\frac{v}{p}\right)^{r}$.

Example 1. Let $b=p q r$ where $p, q$, and $r$ are rational primes with $p, q \equiv 3$ $\bmod 8$ and $r \equiv 7 \bmod 8$, let $\alpha=(b, t)_{\mathbb{Q}(t)}$, let $K=\mathbb{Q}(t)\left(\sqrt{b t^{2}+b}\right)$, and let $\beta=\operatorname{res}_{K / \mathbb{Q}(t)}(\alpha)=(b, t)_{K}$. Then $\beta \neq 0$ but $\rho_{T}(\beta)=0$ for every place $T$ of $K / \mathbb{Q}$ with $T(t) \in \mathbb{Q}_{\text {alg }}-\{0\}$.

Proof. We first note that the norm from $K(\sqrt{b})$ to $K$ of $(b t+b)+\left(\sqrt{b t^{2}+b}\right) \sqrt{b}$ equals $(b t+b)^{2}-b\left(b t^{2}+b\right)=2 t b^{2}$, and so $2 t$ is a norm from $K(\sqrt{b})$ to $K$. Thus $(b, 2 t)_{K}=0$, and so $\beta=(b, t)_{K}=(b, 2)_{K}$.

Suppose first that $\beta$ is trivial. Then $(b, 2)_{K}=0$. But $K$ is a generic splitting field for $(b, b)_{\mathbb{Q}}=(b,-1)_{\mathbb{Q}}$. It follows by [J], Theorem 3.10.1] that $(b, 2)_{\mathbb{Q}}=(b,-1)_{\mathbb{Q}}$. In particular, $(p q r, 2)_{\mathbb{Q}}$ and $(p q r,-1)_{\mathbb{Q}}$ have the same Hasse invariants. Since $r \equiv 7$ $\bmod 8$, we have $(p q r,-1)_{r}=\left(\frac{-1}{r}\right)=-1$ while $(p q r, 2)_{r}=\left(\frac{2}{r}\right)=1$ [We, p. 252], a contradiction. Thus $\beta \neq 0$.

Let $T$ be a place of $K / \mathbb{Q}$ with $T(t)=c \in \mathbb{Q}_{\text {alg }}-\{0\}$ and let $E=\bar{K}_{T}=$ $\mathbb{Q}(c)\left(\sqrt{b\left(c^{2}+1\right)}\right)$. Then $\rho_{T}(\beta)=(b, 2)_{E}$. We will show that $\rho_{T}(\beta)$ is trivial by showing that it has multiplicative Hasse invariant 1 at all primes of $E$.

Since $K$ is a generic splitting field for $(b,-1)_{\mathbb{Q}}, E$ splits $(b,-1)_{\mathbb{Q}}$ [J, p. 123]. Exactly as above, one can show using [We, p. 252] that $(b,-1)_{\mathbb{Q}}$ has multiplicative Hasse invariant -1 precisely at $2, p, q$, and $r$, while $(b, 2)_{\mathbb{Q}}$ has multiplicative Hasse invariant -1 precisely at $p$ and $q$. Since $E$ splits $(b,-1)_{\mathbb{Q}}$, the local degree of every extension of the rational primes $2, p, q$, and $r$ to $E$ is even [P, Lemma 18.4]. But then $E$ also splits $(b, 2)_{\mathbb{Q}}=\rho_{T}(\beta)$, since $(b, 2)_{\mathbb{Q}}$ has multiplicative Hasse invariant -1 precisely at $p$ and $q$. Thus $\rho_{T}(\beta)=0$, as was to be shown.

Remark 4.1. In Example 1, the hypotheses of Corollary 3.2 (Special Case) are not satisfied because $\sqrt{b} \in \mathbb{Q}(\sqrt{g(0)})$. Thus this example shows that we cannot eliminate this hypothesis.

Remark 4.2. The remarkable Brauer class $(b, 2)_{\mathbb{Q}}$ in Example 1 was discovered by Witt [Wi] and was used by him to show that the Hasse norm theorem does not hold for algebraic function fields. The argument that $(b, 2)_{\mathbb{Q}}$ is split by every residue field 
of $K$ appears in [Wi] and is repeated above for the convenience of the reader. The Brauer class $\beta$ of Example 1 is shown in the course of the proof to equal $(b, 2)_{K}$. In particular, $\beta$ is a constant class in the sense that it equals $\operatorname{res}_{K / \mathbb{Q}}\left(\beta_{0}\right)$ for some $\beta_{0} \in \operatorname{Br}(\mathbb{Q})$. Although we will not prove it, it can be shown that our other examples are not constant classes.

Before proceeding further, we prove two preliminary results. We begin with a useful criterion for the specialization of $\beta$ at a place of $K / \mathbb{Q}$ to be non-trivial.

Lemma 4.1. Let $K=\mathbb{Q}(t)(\sqrt{g(t)})$, where $g(t) \in \mathbb{Q}[t]$ is square-free, and let $\alpha=$ $(b, t)_{\mathbb{Q}(t)}$ and $\beta=(b, t)_{K}$, where $b \in \mathbb{Q}-\mathbb{Q}^{2}$. Let $c \in \mathbb{Q}_{\text {alg }}-\{0\}$, and let $T$ be a place of $K$ extending the place $P_{c}$ of $\mathbb{Q}(t) / \mathbb{Q}$ which sends $t \rightarrow c$. Then $\exp \left(\rho_{T}(\beta)\right)=2$ if and only if there exists a valuation $\pi$ of $\mathbb{Q}(c)$ splitting completely in $\mathbb{Q}(c, \sqrt{g(c)})$ such that $(b, c)_{\pi}=-1$.

Proof. Let $E=\bar{K}_{T}=\mathbb{Q}(c, \sqrt{g(c)})$. Then $\rho_{T}(\beta)=(b, c)_{E}=\operatorname{res}_{E / \mathbb{Q}(c)}(b, c)_{\mathbb{Q}(c)}$. By [P, Theorem 18.6 and Corollary 18.6], $\exp \left(\rho_{T}(\beta)\right)=2$ if and only if there exists a valuation $\delta$ of $E$ such that $(b, c)_{\delta}=-1$. By $[\mathrm{P}$, Lemma 18.4], this occurs if and only if there exists a valuation $\pi$ of $\mathbb{Q}(c)$ such that $(b, c)_{\pi}=-1$ and such that $\left[E_{\delta}: \mathbb{Q}(c)_{\pi}\right]$ is odd for some extension $\delta$ of $\pi$ to $E$. Since $[E: \mathbb{Q}(c)] \leq 2$, Lemma 4.1 follows.

Our next preliminary result reduces the problem of finding infinitely many $c$ with the desired properties to that of finding a single one.

Lemma 4.2. Let $K=\mathbb{Q}(t)(\sqrt{g(t)})$, where $g(t) \in \mathbb{Q}[t]$ is square-free, and let $\alpha=$ $(b, t)_{\mathbb{Q}(t)}$ and $\beta=(b, t)_{K}$, where $b \in \mathbb{Q}-\mathbb{Q}^{2}$. Assume that there exists a $d \in$ $\mathbb{Q}_{\text {alg }}-\{0\}$ such that the place $P_{d}$ of $\mathbb{Q}(t) / \mathbb{Q}$ sending $t \rightarrow d$ has an extension $Q$ to $K$ with $\rho_{Q}(\beta) \neq 0$. Then there exist infinitely many places $T$ of $K / \mathbb{Q}$ with $T(t) \in \mathbb{Q}_{\text {alg }}-\{0\}$ and $\exp \left(\rho_{T}(\beta)\right)=2$; moreover, if $d \in \mathbb{Q}$, then these infinitely many $T$ may be chosen so that $T(t) \in \mathbb{Q}$.

Proof. Let $E=\bar{K}_{Q}=\mathbb{Q}(d)(\sqrt{g(d)})$. By assumption, $(b, d)_{E} \neq 0$. By Lemma 4.1 , there exists a valuation $\pi$ of $\mathbb{Q}(d)$ such that $\pi$ splits completely in $E$ and $(b, d)_{\pi}=-1$. In particular, $g(d)$ is a square in $\mathbb{Q}(d)_{\pi}$.

Let $h(t)=g(t)$ if $g(d) \neq 0$ and $h(t)=g(t) /(t-d)$ if $g(d)=0$. Since $g(t)$ is square-free, $h(d) \neq 0$. We claim that if $v \in \mathbb{Q}$ is chosen close enough to $0 \pi$-adically and $c=d+h(d) v^{2}$, then the place $P_{c}$ of $\mathbb{Q}(t) / \mathbb{Q}$ has an extension $T$ to $K$ with $\exp \left(\rho_{T}(\beta)\right)=2$. Exactly how close $v$ needs to be taken to $0 \pi$-adically will become clear in the course of the proof.

Our first requirement is that $v$ should be chosen close enough to $0 \pi$-adically so that $c d^{-1}=1+h(d) d^{-1} v^{2}$ is a square in $\mathbb{Q}(d)_{\pi}$. It is clear that this is possible whether $\pi$ is a finite or infinite prime.

Our second requirement is that $v$ be chosen close enough to $0 \pi$-adically so that $g(c)$ is a square in $\mathbb{Q}(d)_{\pi}$. To see that this is possible, recall that $g(d)$ is a square in $\mathbb{Q}(d)_{\pi}$. Suppose first that $g(d) \neq 0$. Then any element of $\mathbb{Q}(d)_{\pi}$ sufficiently close to $g(d)$ will also be a square. Since polynomials are continuous functions, if $c$ is close enough $\pi$-adically to $d$, then $g(c)$ will be close enough $\pi$-adically to $g(d)$ to insure that $g(c)$ is a square in $\mathbb{Q}(d)_{\pi}$. Since $c=d+h(d) v^{2}$, it is clear that $c$ can be brought close enough to $d \pi$-adically so that $g(c)$ is a square in $\mathbb{Q}(d)_{\pi}$. Suppose next that $g(d)=0$. In this case, $g(t)=(t-d) h(t)$. Since $c=d+h(d) v^{2}$, we have 


$$
\begin{aligned}
g(c) & =(c-d) h(c)=h(d) v^{2} h(c) \\
& =h(d) v^{2}((h(c)-h(d))+h(d))=(h(d) v)^{2}+h(d)\left[h\left(d+h(d) v^{2}\right)-h(d)\right] v^{2} .
\end{aligned}
$$

Since $h(d)\left[h\left(d+h(d) v^{2}\right)-h(d)\right] v^{2}$ can be brought arbitrarily close to $0 \pi$-adically by a suitable choice of $v$, it is clear that $g(c)$ can be brought arbitrarily close $\pi$ adically to $(h(d) v)^{2}$. Thus $g(c)$ will be a square in $\mathbb{Q}(d)_{\pi}$ for $v$ sufficiently close to $0 \pi$-adically.

Now let $v \in \mathbb{Q}$ be chosen close enough to $0 \pi$-adically so that $c d^{-1}$ and $g(c)$ are both squares in $\mathbb{Q}(d)_{\pi}$. Let $T$ be any extension of $P_{c}$ to $K$. We will show that $\exp \left(\rho_{T}(\beta)\right)=2$.

Since $c=d+h(d) v^{2}$ and $v \in \mathbb{Q}, \mathbb{Q}(c) \subseteq \mathbb{Q}(d)$. Let $L=\mathbb{Q}(c)(\sqrt{g(c)})$, and let $M=\mathbb{Q}(d)(\sqrt{g(d)}, \sqrt{g(c)})$. Let $\delta$ be an extension of $\pi$ to $M$ and let $\theta$ denote the restriction of $\delta$ to $L$. Since $\rho_{T}(\beta)=(b, c)_{L}$, it suffices to show that $(b, c)_{\theta}=-1$. Since $(b, c)_{\delta}=\left((b, c)_{\theta}\right)^{r}$, where $r=\left[M_{\delta}: L_{\theta}\right]$, it suffices to show that $(b, c)_{\delta}=-1$. Since $c d^{-1}$ is a square in $\mathbb{Q}(d)_{\pi},(b, c)_{\pi}=(b, d)_{\pi}=-1$. Since $g(c)$ and $g(d)$ are both squares in $\mathbb{Q}(d)_{\pi}, M_{\delta}=\mathbb{Q}(d)_{\pi}$. Thus $(b, c)_{\delta}=(b, c)_{\pi}=-1$, as was to be shown.

Our next example shows that we cannot eliminate the hypothesis that $g(0) \neq 0$ in Corollary 3.2 (Special Case). In particular, this shows that Theorem 3.1 does not hold if the hypothesis that $P_{i}$ is unramified in $K$ for $1 \leq i \leq u$ is eliminated.

Example 2. Let $q$ be a rational prime with $q \equiv 3 \bmod 8$, let $\alpha=(2 q, t)_{\mathbb{Q}(t)}$, let $K=\mathbb{Q}(t)\left(\sqrt{q t\left(t^{4}+1\right)}\right)$, and let $\beta=(2 q, t)_{K}$. Then:

(i) $\beta \neq 0$,

(ii) $\rho_{T}(\beta)=0$ for every place $T$ of $K / \mathbb{Q}$ with $T(t) \in \mathbb{Q}-\{0\}$, and

(iii) there exist infinitely many places $U$ of $K / \mathbb{Q}$ with $U(t) \in \mathbb{Q}_{\text {alg }}-\{0\}$ and $\exp \left(\rho_{U}(\beta)\right)=2$.

Proof. Let $\zeta$ be a primitive 8-th root of unity and let $S$ be a place of $K / \mathbb{Q}$ extending $P_{\zeta}$. Then $\bar{K}_{S}=\mathbb{Q}(\zeta) . \quad \rho_{S}$ is defined on $\beta$, and $\rho_{S}(\beta)=(2 q, \zeta)_{\mathbb{Q}(\zeta)}$. The $q$-adic valuation of $\mathbb{Q}$ has two extensions to $\mathbb{Q}(\zeta)$, each unramified of degree 2 . Let $\tau$ be one of these extensions. Then $2 q$ is a uniformizer for $\tau$, and $\tau$ is unramified of degree 2 in $\mathbb{Q}(\sqrt{\zeta})$. It follows that $\operatorname{inv}_{\tau}\left(\rho_{S}(\beta)\right)=-1$. Thus $\rho_{S}(\beta) \neq 0$, and so $\beta \neq 0$. By Lemma 4.2 , there exist infinitely many $c \in \mathbb{Q}_{\text {alg }}-\{0\}$ such that $P_{c}$ has an extension $U$ to $K$ with $\exp \left(\rho_{U}(\beta)\right)=2$. This proves (i) and (iii).

Suppose next that $T$ is a place of $K / \mathbb{Q}$ with $T(t)=c \in \mathbb{Q}-\{0\}$ and with $\left.\exp \left(\rho_{T}\right)(\beta)\right)=2$. Let $E=\bar{K}_{T}=\mathbb{Q}\left(\sqrt{q c\left(c^{4}+1\right)}\right)$. Then $\rho_{T}(\beta)=(2 q, c)_{E}$. Since $\exp \left(\rho_{T}(\beta)\right)=2$ by assumption, there exists a prime $\pi$ of $E$ such that $\operatorname{inv}_{\pi}\left(\rho_{T}(\beta)\right)=$ -1 . Since $2 q>0, \pi$ is a finite prime. Let $\pi_{\mathbb{Q}}$ be equivalent to the $p$-adic valuation of $\mathbb{Q}$. We will obtain a contradiction by eliminating all possibilities for $p$.

Let $\rho_{c}(\alpha)=(2 q, c)_{\mathbb{Q}}$. Since $\rho_{T}(\beta)=\operatorname{res}_{E / \mathbb{Q}}\left(\rho_{c}(\alpha)\right)$, it follows that $(2 q, c)_{p}=-1$ and $p$ splits completely in $E$. In particular, $q c\left(c^{4}+1\right)$ is a square in $\mathbb{Q}_{p}$.

Suppose first that $p$ is odd, $p \neq q$. The $p$-adic valuation of $c$ must be odd, since $(2 q, c)_{p}=-1$. But then the $p$-adic valuation of $q c\left(c^{4}+1\right)$ is also odd, and so $p$ ramifies in $E$, a contradiction. Thus $p$ is either 2 or $q$.

We observe next that $(2 q, q)_{q}=(2 q, q)_{2}=1$. It is clear that the only non-trivial Hasse invariants of $(2 q, q)_{\mathbb{Q}}$ can be at 2 and $q$. It follows from the Hasse sum formula that $(2 q, q)_{2}=(2 q, q)_{q}$. But $(2 q, q)_{2}=(2, q)_{2}(q, q)_{2}=(2, q)_{2}(-1, q)_{2}=(-1)(-1)=$ 1 since $q \equiv 3 \bmod 8$. Thus $(2 q, q)_{q}=(2 q, q)_{2}=1$. 
Now suppose that $p=q$. The $p$-adic valuation of $c$ must be odd, since otherwise the $p$-adic valuation of $q c\left(c^{4}+1\right)$ is odd and so $p$ ramifies in $E$, a contradiction. In particular, $1+c^{4}$ is a square in $\mathbb{Q}_{p}$. Since $q c\left(c^{4}+1\right)$ is also known to be a square in $\mathbb{Q}_{p}, q c$ is a square in $\mathbb{Q}_{p}$. Let $c=q^{r} z$, where $z$ is a $q$-unit and $r$ is odd. Then $q^{r+1}, 1+c^{4}$, and $q c$ are all squares in $\mathbb{Q}_{p}$, and since $q^{r+1} z\left(c^{4}+1\right)=q c\left(c^{4}+1\right), z$ is a square in $\mathbb{Q}_{p}$. But then $(2 q, c)_{q}=\left(2 q, q^{r} z\right)_{q}=(2 q, q)_{q}=1$, a contradiction.

Finally, suppose that $p=2$. Let $c=2^{r} z w^{2}$, where $z$ is a square-free rational number and $z$ and $w$ are 2-units. If $r=0$ or is odd, then the 2-adic valuation of $q c\left(c^{4}+1\right)$ is also odd, so 2 is ramified in $E$, a contradiction. Thus $r$ is even, $r \neq 0$, so $1+c^{4}$ is a square in $\mathbb{Q}_{2}$. Since $q c\left(c^{4}+1\right)$ is known to be a square in $\mathbb{Q}_{2}$, $q c$ is a square in $\mathbb{Q}_{2}$ and so $(2 q, q c)_{2}=1$. But, as shown above, $(2 q, q)_{2}=1$ and so $1=(2 q, q c)_{2}=(2 q, q)_{2}(2 q, c)_{2}=(2 q, c)_{2}$, a contradiction. This eliminates all possibilities for $p$, and shows that $c$ and $T$ cannot exist.

Remark 4.3. Examples 1 and 2 show that, in Corollary 3.2 (Special Case), we cannot eliminate either the hypothesis that $g(0) \neq 0$ or the hypothesis that $\sqrt{b} \notin$ $\mathbb{Q}(\sqrt{g(0)})$. One difference between the two examples, however, is that in Example $2, \rho_{U}(\beta) \neq 0$ for infinitely many specializations $U$ of $K / \mathbb{Q}$ with $U(t) \in \mathbb{Q}_{\text {alg }}-\{0\}$ which are defined on $\beta$, while in Example 1 no such $U$ exist. We remark that there exist examples similar to Example 2 where $g(0) \neq 0$, which show that the hypothesis that $\sqrt{b} \notin \mathbb{Q}(\sqrt{g(0)})$ cannot be eliminated. Let $p$ and $q$ be primes with $p \equiv 3 \bmod 8$ and $q \equiv 7 \bmod 8$. Let $b=2 p q$ and $g(t)=b t^{4}+b$. Let $\alpha=(b, t)_{\mathbb{Q}(t)}$, $K=\mathbb{Q}(t)\left(\sqrt{b t^{4}+b}\right)$, and $\beta=\operatorname{res}_{K / \mathbb{Q}(t)}(\alpha)$. Arguing almost exactly as in the proof of Example 2, one can show that $\beta \neq 0$, that for each $c \in \mathbb{Q}-\{0\}$ and each extension $T$ of $P_{c}$ to $K$ we have $\rho_{T}(\beta)=0$, and that there exist infinitely many $c \in \mathbb{Q}_{\text {alg }}-\{0\}$ such that $P_{c}$ has an extension $U$ to $K$ with $\exp \left(\rho_{U}(\beta)\right)=2$. We omit the routine verification.

Remark 4.4. The examples we have presented have been of exponent 2. There do exist, however, examples similar to Example 2 but of larger exponent. Let $\omega$ be a primitive cube root of unity and let $F=\mathbb{Q}(\omega)$. Let $p$ and $q$ be rational primes with $p \equiv 1 \bmod 27, q \equiv 1 \bmod 3$, and with $q$ not a cube modulo $p$. Let $\alpha$ be the Brauer class containing the $F(t)$-algebra generated by $x$ and $y$ subject to the relations $x^{3}=p, y^{3}=q$, and $x y=\omega y x$. Let $K=F(t)\left(\sqrt[3]{q t^{3}+p}\right)$ and let $\beta=\operatorname{res}_{K / F(t}(\alpha)$. To show that $\beta \neq 0$, let $\pi$ be the prime of $F(\sqrt[3]{q})$ extending the rational prime $p$, and let $n$ be maximal such that $F(\sqrt[3]{q})_{\pi}$ contains a $3^{n}$-th root of $q$. Let $c$ be a $3^{n}$-th root of $q$ and let $T$ be a place of $K / F$ extending the place $P_{c}$ of $F(t) / F$. Then $\operatorname{inv}_{\pi}\left(\rho_{T}(\beta)\right)$ is non-trivial, and so $\beta$ is also non-trivial. A tedious argument similar to that used in Example 2 shows that there do not exist any $c \in F$ such that $P_{c}$ has an extension to a place $S$ of $K / F$ with $\rho_{S}(\beta)$ non-trivial.

Our final example shows that the hypotheses of Corollary 3.2 (Special Case) are not necessary and sufficient.

Example 3. Let $b \in \mathbb{Q}-\mathbb{Q}^{2}$, let $a \in \mathbb{Q}-\{0\}$, let $\alpha=(b, t)_{\mathbb{Q}(t)}$, let $K=$ $\mathbb{Q}(t)\left(\sqrt{a t^{2}+b}\right)$, and let $\beta=\operatorname{res}_{K / \mathbb{Q}(t)}(\alpha)=(b, t)_{K}$. Assume that $\mathbb{Q}(\sqrt{a}) \neq \mathbb{Q}(\sqrt{b})$. Then $\beta \neq 0$, and there exist infinitely many $c \in \mathbb{Q}-\{0\}$ such that the place $P_{c}$ of $\mathbb{Q}(t) / \mathbb{Q}$ sending $t \rightarrow c$ has an extension to a place $T$ of $K / \mathbb{Q}$ with $\exp \left(\rho_{T}(\beta)\right)=2$.

Proof. Let $\sigma$ generate $\operatorname{Gal}(\mathbb{Q}(\sqrt{a}, \sqrt{b}) / \mathbb{Q}(\sqrt{a})$. By the Tchebotarev density theorem [FJ p. 58] there exists an odd prime $p$ of $\mathbb{Q}$ such that $p$ does not divide $a b$, 
$p$ is unramified in $\mathbb{Q}(\sqrt{a}, \sqrt{b})$, and such that $\sigma$ is the Frobenius automorphism at $p$. In particular, $p$ splits completely in $\mathbb{Q}(\sqrt{a})$ but not in $\mathbb{Q}(\sqrt{b})$, and so $\left(\frac{a}{p}\right)=1$ and $\left(\frac{b}{p}\right)=-1$. Let $c=1 / p$. Then $(b, c)_{p}=(b, p)_{p}=-1$, since $\left(\frac{b}{p}\right)=-1$. Also, $\mathbb{Q}_{p}\left(\sqrt{a c^{2}+b}\right)=\mathbb{Q}_{p}\left(\sqrt{a+b p^{2}}\right)=\mathbb{Q}_{p}(\sqrt{a})=\mathbb{Q}_{p}$, since $\left(\frac{a}{p}\right)=1$. Let $T$ be an extension of $P_{c}$ to $K$. By Lemma 4.1, $\exp \left(\rho_{T}(\beta)\right)=2$. By Lemma 4.2 there are infinitely many choices of $c \in \mathbb{Q}$ for which this holds. Since $\exp \left(\rho_{T}(\beta)\right)=2$, $\exp (\beta)=2$.

Remark 4.5. The reader may wonder what can happen in Example 3 if $\mathbb{Q}(\sqrt{a})=$ $\mathbb{Q}(\sqrt{b})$. Example 1 illustrates that one can have $\beta \neq 0$, but this cannot be shown by a specialization argument with $t \rightarrow c \in \mathbb{Q}_{\text {alg }}-\{0\}$. It is worth noting that one can also have $\beta=0$. Consider the case when $a=b=3$. Then $\alpha=(3, t)_{\mathbb{Q}(t)}$, $\left.K=\mathbb{Q}(t)\left(\sqrt{3 t^{2}+3}\right)\right)$, and $\beta=(3, t)_{K}$. Let $\{1, i, j, k\}$ be the standard basis for $(3, t)_{\mathbb{Q}(t)}$, so $i^{2}=3, j^{2}=t$, and $i j=k=-j i$. Then $[(t-1) i+3 j+k]^{2}=3 t^{2}+3$. Thus $K$ splits $\alpha$, and so $\beta=0$. (We are indebted to Adrian Wadsworth for this simple argument.)

\section{REFERENCES}

[E] O. Endler, Valuation theory, Springer-Verlag, Berlin/Heidelberg/New York, 1972. MR 50:9847

[FSS] B. Fein, D. Saltman, and M. Schacher, Brauer-Hilbertian fields, Trans. Amer. Math. Soc. 334 (1992), 915-928. MR 93b:12006

[FS1] B. Fein and M. Schacher, Brauer groups of rational function fields over global fields, Groupe de Brauer (M. Kervaire and M. Ojanguren, eds.), LNM\#844, Springer-Verlag, Berlin 1981, pp. 46-74. MR 82h:12025

[FS2] - A conjecture about relative Brauer groups, in K-Theory and Algebraic Geometry: Connections with Quadratic Forms and Division Algebras (B. Jacob and A. Rosenberg, eds.), Proceedings of Symposia in Pure Mathematics, Vol. 58, Amer. Math. Soc., Providence, pp. 161-169. MR 96e:12006

[FS3] Crossed products over algebraic function fields, J. Algebra 171 (1995), 531-540. MR 96b:12003

[FJ] M. Fried and M. Jarden, Field arithmetic, Springer-Verlag, Berlin, 1986. MR 89b:12010]

[G] W.-D. Geyer, Galois groups of intersections of local fields, Israel J. of Mathematics 30 (1978), 382-396. MR 80a:12017]

[J] N. Jacobson, Finite-dimensional division algebras over fields, Springer-Verlag, Berlin, 1996. MR 98a:16024]

[P] R. Pierce, Associative algebras, Springer-Verlag, Berlin, 1982. MR 84c:16001

[Sa] D. Saltman, The Brauer group and the center of generic matrices, J. Algebra 97 (1985), 53-67. MR 87a:13005

[Se1] J.-P. Serre, Corps locaux, Hermann, Paris, 1962. MR 27:133

[Se2] J.-P. Serre, Spécialisation des éléments de $\operatorname{Br}_{2}\left(\mathbb{Q}\left(T_{1}, \ldots, T_{n}\right)\right)$, C. R. Acad. Sci. Paris 311 (1990), 397-402. MR 91m:12007

[V] I. I. Voronovich, Linear local-global principle for algebras over rational function fields, Preprint \#25(295), Minsk, 1987 (Russian).

[We] E. Weiss, Algebraic Number Theory, Mc-Graw Hill, New York, 1963. MR 28:3021

[Wi] E. Witt, Gegenbeispiel zum Normensatz, Math. Zeit. 39 (1934), 462-467.

Department of Mathematics, Oregon State University, Corvallis, Oregon 97331

E-mail address: fein@math.orst.edu

Department of Mathematics, University of California at Los Angeles, Los Angeles, CALIFORNIA 90024

E-mail address: mms@math.ucla.edu 